\title{
Magnetic wave energy fluxes for late-type stars
}

\section{Longitudinal tube waves}

\author{
P. Ulmschneider ${ }^{1}$, Z. E. Musielak ${ }^{2,1}$, and D. E. Fawzy ${ }^{1}$ \\ 1 Institut für Theoretische Astrophysik der Universität Heidelberg, Tiergartenstr. 15, 69121 Heidelberg, Germany \\ 2 Department of Physics, University of Texas at Arlington, Arlington, TX 76019, USA
}

Received 17 July 2000 / Accepted 10 May 2001

\begin{abstract}
The wave energy fluxes carried by longitudinal tube waves along thin vertical magnetic flux tubes embedded in atmospheres of late-type stars are computed. The main physical process responsible for the generation of these waves is the nonlinear time-dependent response of the flux tubes to continuous and impulsive external turbulent pressure fluctuations, which are represented here by an extended Kolmogorov spatial and modified Gaussian temporal energy spectrum. Both the wave energy fluxes and spectra are calculated for population I stars with effective temperatures ranging from $T_{\text {eff }}=3500 \mathrm{~K}$ to $7000 \mathrm{~K}$, and with gravities in the range $\log g=3-5$. The obtained results show that the computed wave energy may significantly contribute to the enhanced heating observed in magnetic regions of late-type stars.
\end{abstract}

Key words. methods: numerical - stars: chromospheres - stars: coronae - stars: magnetic fields - MHD

\section{Introduction}

It has become clear in recent years that acoustic and magnetic waves generated in stellar convection zones play a dominant role in the heating of stellar chromospheres, and may also contribute significantly to the heating of stellar coronae as well as to the acceleration of stellar winds (see Narain \& Ulmschneider 1996). Our recent work has shown that previously calculated stellar acoustic wave energy fluxes (e.g., Bohn 1981, 1984) are incorrect, mainly because several fundamental assumptions in the LighthillStein theory (Lighthill 1952; Stein 1967) of sound generation turned out to be physically unjustified (Musielak et al. 1994). We have corrected the Lighthill-Stein theory by incorporating an improved description of the spatial and temporal spectrum of the turbulent convection and used this corrected theory to calculate new stellar acoustic wave energy fluxes (see Ulmschneider et al. 1996 for stars of solar type metal abundances; and Ulmschneider et al. 1999, for stars with nonsolar metallicities). That work focused on the generation of acoustic waves, and did not involve stellar magnetic fields. Given that stars have internal and surface magnetic fields, we have also worked on the complementary problem of the excitation of waves in magnetic flux tubes. Specifically, we have concentrated on the generation of longitudinal and transverse tube waves, and developed general analytical (Musielak et al. 1989,

Send offprint requests to: P. Ulmschneider,

e-mail: ulmschneider@ita.uni-heidelberg.de
1995) and numerical (Huang et al. 1995; Ulmschneider \& Musielak 1998) treatments to describe this process.

In the present paper, we use the approach developed by Ulmschneider \& Musielak (1998) to compute the wave energy fluxes carried by longitudinal tube waves propagating along thin and vertically oriented magnetic flux tubes that are embedded in atmospheres of late-type stars. This numerical approach supplements recent work by Musielak et al. (2000), who calculated analytically the longitudinal wave energy fluxes generated in stellar convection zones. These newly calculated acoustic and magnetic wave energy fluxes are essential for constructing theoretical chromospheric models of stars of different spectral types and luminosities (see Buchholz et al. 1998; also Cuntz et al. 1998, 1999).

In the numerical approach developed by Ulmschneider \& Musielak (1998), longitudinal tube waves are generated as a result of the squeezing of a thin and vertically oriented magnetic flux tube by external pressure fluctuations produced by the turbulent motions in a stellar photosphere and convection zone. Hence, to compute the pressure fluctuations imposed on the tube, it is required to know the external turbulent motions. The motions are modeled by specifying the rms velocity amplitude and using an extended Kolmogorov turbulent energy spectrum with a modified Gaussian frequency factor (Musielak et al. 1994). It is assumed that the squeezing is symmetric with respect to the tube axis. The computed pressure fluctuations are then translated into the gas pressure and magnetic field 
fluctuations inside the tube by using the horizontal pressure balance. Finally, the internal velocity perturbation resulting from the internal pressure fluctuation is calculated. This internal velocity serves as a boundary condition for a numerical longitudinal tube wave code.

That code used by Ulmschneider \& Musielak (1998) was originally developed by Herbold et al. (1985) who treated magnetic flux tubes in the so-called thin flux tube approximation and described them mathematically by using a set of one-dimensional, time-dependent and nonlinear MHD equations. The code allows to compute the instantaneous and time-averaged longitudinal tube wave energy fluxes and the corresponding wave energy spectra. It requires specifying the strength of the magnetic field inside the flux tube and the height in the stellar atmosphere where the squeezing takes place. The code has been extensively used to calculate wave energy fluxes and spectra for longitudinal tube waves propagating in the solar atmosphere, and to investigate the dependence of these fluxes on the magnetic field strength, the rms velocity amplitude of turbulent motions, and the location of the squeezing in the atmosphere. A modified version of this code has also been used to study the propagation of longitudinaltransverse magnetic tube waves in the solar atmosphere (Ulmschneider et al. 1991) and the generation of transverse tube waves in the solar photosphere and convection zone (Huang et al. 1995).

In the approach developed by Ulmschneider \& Musielak (1998), the external pressure fluctuations are represented by a superposition of many partial waves with random phases and with amplitudes determined by the turbulent flow field. It has been shown that occasionally the amplitude of this superposition of waves become comparable to the horizontal velocities observed on the Sun at the photospheric level (Muller 19; Nesis et al. 1992; Muller et al. 1994) and also seen in time-dependent numerical simulations of solar convection (e.g., Nordlund \& Dravins 1990; Nordlund \& Stein 1991; Cattaneo et al. 1991; Steffen 1993; see also Nordlund et al. 1997). These more realistic velocity fluctuations are clearly a main advantage of our approach, and Ulmschneider \& Musielak have used this argument to justify their studies of the generation of nonlinear longitudinal tube waves in the solar atmosphere.

The main goal of the present paper is to extend these studies to other late-type stars and compute the wave energy fluxes and spectra for population I stars with effective temperatures ranging from $T_{\text {eff }}=3500 \mathrm{~K}$ to $7000 \mathrm{~K}$, and with gravities in the range $\log g=3-5$. The calculated amount of wave energy carried by nonlinear longitudinal tube waves in atmospheres of K-type stars has already been used by Cuntz et al. (1999) to construct theoretical, time-dependent and self-consistent chromospheric models for these stars. Our paper is organized as follows: Sect. 2 describes briefly the method developed by Ulmschneider and Musielak and our basic assumptions. The results of our calculations are presented in Sect. 3, and finally Sect. 4 gives our conclusions.

\section{Basic formulation}

\subsection{Convection zone models and turbulent velocities}

The method to calculate wave energy fluxes carried by longitudinal tube waves adopted in the present paper has been described by Ulmschneider \& Musielak (1998), thus, it is not necessary to repeat a detailed discussion here. In the solar application, we were able to select many parameters and characteristic values directly from observations. However, for other stars such data are not available. Therefore, in the following we first discuss in greater detail the physical reasoning behind our choice of several parameters used in the calculations and then briefly summarize the adopted computational method.

Both numerical simulations of stellar convection and mixing length models show that the maximum convective velocities occur at optical depths of $\tau_{5000} \approx 10$ to 100 . For example, Steffen (1993) found in his time-dependent solar numerical convection calculations that maximum convective velocities $v_{\mathrm{CMax}} \approx 2.8 \mathrm{~km} \mathrm{~s}^{-1}$ are reached at $\tau_{5000} \approx 50$ and that these values can be reproduced by the mixing length theory with a mixing length parameter of $\alpha \approx 2$. This choice of $\alpha=2$ is also indicated by timedependent hydrodynamic simulations of stellar convection of stars other than the Sun (Trampedach et al. 1997) as well as by a careful fitting of evolutionary tracks of the Sun with its present luminosity, effective temperature and age (Hünsch \& Schröder 1997; Schröder \& Eggleton 1996).

Our treatment of stellar convection zones is similar to that described by Ulmschneider et al. (1996). We also take $\alpha=2$ and use a solar metal abundance. In Fig. 1, we plot the ratio of the maximum convective velocity to the local speed of sound (the so-called convective Mach number) for stars of different spectral types and luminosities. It is seen that this ratio approaches (or even exceeds) unity for hot and luminous stars. Because the values of the convective Mach numbers higher than one are unrealistic, in this paper we restrict our calculations to stars with gravities ranging from $\log g=5$ to $\log g=3$, and with $T_{\text {eff }}=7000 \mathrm{~K}$ or cooler.

In our approach, however, we actually need to know the velocities of turbulent motions in the overshooting layer near the stellar surface, where the squeezing of the magnetic flux tube takes place. Steffen's numerical calculations show that the rms velocities decrease towards the solar surface and reach a plateau in the overshooting layer. Between $\tau_{5000}=1$ and $10^{-4}$, he finds values of $v_{\text {rms }}=1.4 \mathrm{~km} \mathrm{~s}^{-1}$, which are practically independent of height. Ulmschneider \& Musielak (1998) adopted for the Sun a variety of observed rms velocity amplitudes $u_{\mathrm{t}}$ in the range $0.9<u_{\mathrm{t}}<1.9 \mathrm{~km} \mathrm{~s}^{-1}$, and showed the dependence of the computed fluxes on this velocity.

For stars, these velocities cannot be determined from observations, thus, we assume that the rms velocity fluctuations at the squeezing point are given by $u_{\mathrm{t}}=v_{\mathrm{CMax}} / 2$. The value of $v_{\mathrm{CMax}}$ is evaluated from stellar convection zone models based on the mixing length theory with 


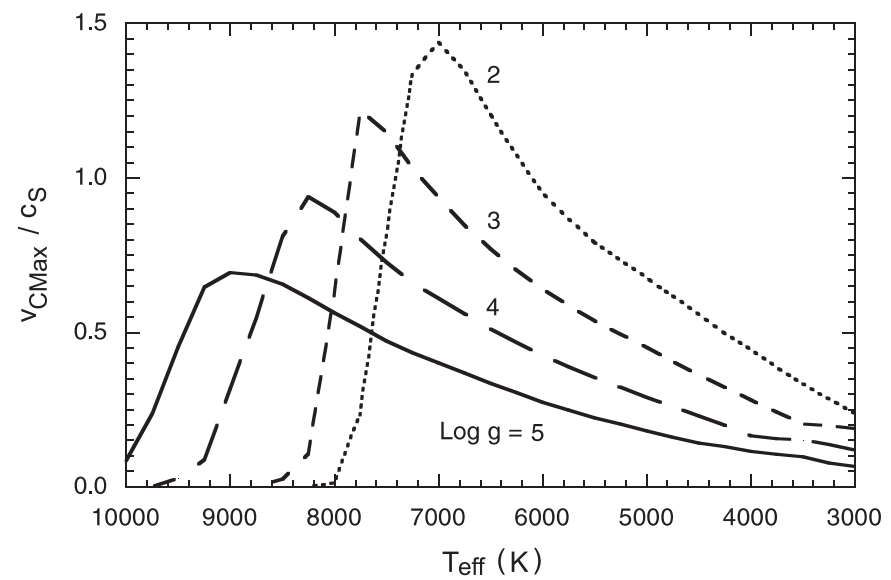

Fig. 1. The ratio of the maximum convective velocity, $v_{\mathrm{CMax}}$, to the local sound speed, $c_{\mathrm{S}}$, is plotted for stars of different spectral types and luminosities.

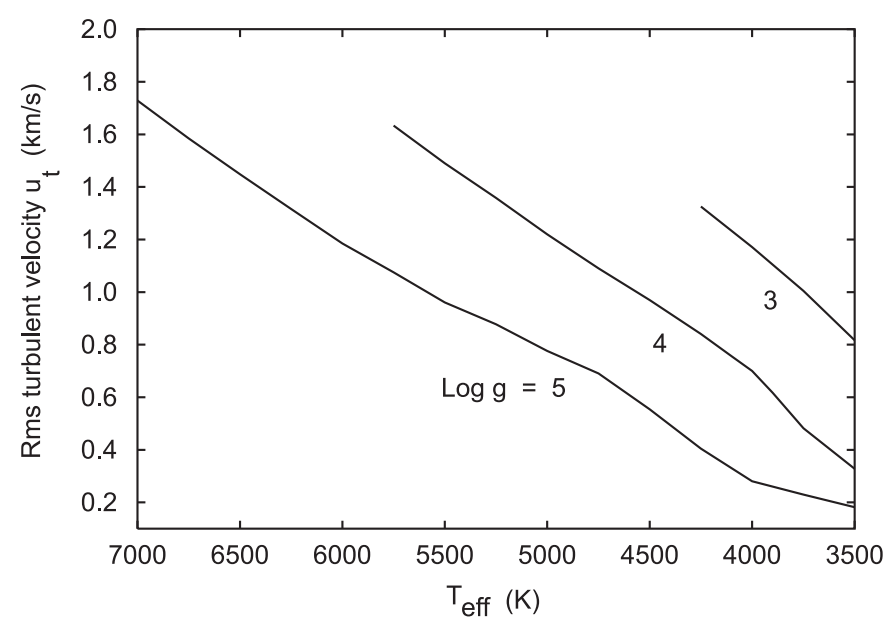

Fig. 2. Root mean square turbulent velocities $u_{\mathrm{t}}=v_{\mathrm{CMax}} / 2$ for late-type stars considered in this paper.

$\alpha=2.0$ (see Fig. 1). The numerical factor 2 used in our calculations assures that in our approach the considered convective velocities are always much lower than the local speed of sound. The values of $u_{\mathrm{t}}=v_{\mathrm{CMax}} / 2$ for the latetype stars considered in this paper are shown in Fig. 2.

After specifying the rms velocities that are responsible for the wave generation, we now determine the height in stellar atmospheres, where the most efficient squeezing of magnetic flux tubes takes place. We guide ourselves by studies performed by Ulmschneider \& Musielak (1998), who pointed out that shifting the height of the excitation point did not change much the resulting wave energy fluxes for the Sun. Based on these results, we take the squeezing point to be located at optical depth $\tau_{5000}=1$ for all considered stars. This depth is commonly taken as the zero height level in stellar atmosphere computations.

The fact that we take only one excitation point in our calculations means that the present method does not take into account the cancellation and amplification (correla- tion) effects, which occur when the flux tube is excited at many points along its length. In reality the tube suffers from squeezing along its entire length directly exposed to the external turbulent motions and, as a result, correlation effects may be important. In the analytical approach developed by Musielak et al. (1989, 1995, 2000), the correlation effects are indeed taken into account but that approach is restricted to linear waves. Our numerical treatment in the present paper does not include the correlation effects, yet, differently from the analytic approach it allows for nonlinear waves.

\subsection{Stellar magnetic flux tubes}

As already mentioned above, the main aim of this paper is to calculate the wave energy fluxes and spectra for longitudinal tube waves propagating along a thin and vertically oriented magnetic flux tube embedded in otherwise magnetic field-free atmospheres of stars with effective temperatures ranging from $T_{\text {eff }}=3500 \mathrm{~K}$ to $7000 \mathrm{~K}$, and with gravities in the range $\log g=3-5$; note that only stars of population I are considered in this paper. To perform these calculations, we must extend the well-known and commonly accepted concept of solar magnetic flux tubes (e.g., Stenflo 1978; Solanki 1993) to stellar flux tubes. At this point, it is important to emphasize that previous (see Saar 1996, and references therein) and recent (e.g., Ruedi et al. 1997) measurements of stellar magnetic fields provide evidence for the existence of strong magnetic fields (typical observed fields are comparable to or stronger than the equipartition field, where equipartition here means equality of the magnetic pressure and the external gas pressure). These fields are characteristic for starspots but not for typical single magnetic flux tubes. Thus, to construct models of stellar magnetic flux tubes, we need to be guided by the results of solar observations. We have to extrapolate these results to stars other than the Sun. Our extrapolation is justified by the fact that the considered stars because of their surface convection zones are fairly similar to the Sun.

In the present paper, we study the efficiency of the generation of longitudinal tube waves in stellar atmospheres. The calculations are performed for one single magnetic flux tube, which means that we are not interested in the filling factor and its consequence for stellar wave luminosities (see, however, Musielak et al. 1995) and for the lower or higher merging at canopy heights of the diverging flux tubes. We follow Ulmschneider \& Musielak (1998) to construct a model of an isolated magnetic flux tube. The most critical physical parameter for this model is the strength of the tube magnetic field, $B$, at the excitation height.

As already discussed by Musielak et al. (1989, 1995), the efficiency of the generation of longitudinal tube waves increases when the "stiffness" of the tube is decreased (or the gas pressure $p$ inside the tube is increased) by decreasing the strength of the tube magnetic field (see also Ulmschneider \& Musielak 1998). Thus, the choice 
of the tube field strength is important. Unfortunately, there are no stellar observations that would allow us to estimate the value of plasma $\beta=8 \pi p / B^{2}$ for stellar magnetic flux tubes. Similarly, there are no fully time-dependent radiation-magnetohydrodynamic simulations of stellar magnetic flux tubes that for our range of stars would supply the value of $B$ and the amount of gas remaining in these tubes.

As a result, we must proceed by analogy to the Sun. A tube, completely devoid of gas, but in pressure equilibrium with the outside gas would have an equipartition field strength $B_{\text {eq }}^{2} / 8 \pi=p_{\mathrm{e}}$, where $p_{\mathrm{e}}$ is the gas pressure outside the tube. Magnetic flux tubes at the solar surface typically have field strengths of the order of $B=1500 \mathrm{G}$.

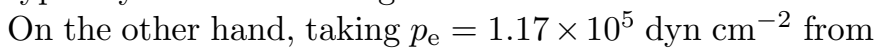
model $\mathrm{C}$ of Vernazza et al. (1981) at the height $z=0$ where $\tau_{5000}=1$, one gets an equipartition field strength of $B_{\text {eq }}=1716 \mathrm{G}$. We thus find a ratio $B / B_{\text {eq }}=0.875$ and consider it as typical for solar and stellar flux tubes. Since this ratio is likely to vary even on the Sun (e.g., Solanki 1993), we have decided to consider three different field strengths, namely, $B / B_{\text {eq }}=0.75,0.85,0.95$ for our stellar flux tubes. This choice of the tube magnetic field has two main advantages: first, after the correct field strength is known, the appropriate longitudinal wave flux can simply be interpolated and second, the dependence of the longitudinal wave flux on the field strength can be investigated.

\subsection{Wave energy fluxes and spectra}

The external pressure fluctuations responsible for squeezing a thin and vertically oriented magnetic flux tube are assumed to be caused by external velocity fluctuations, which are modeled by the superposition of a large number $(N=500)$ of partial waves

$v_{x}(t)=\sum_{n=1}^{N} u_{n} \sin \left(\omega_{n} t+\varphi_{n}\right)$,

where $u_{n}$ is the velocity amplitude of these waves, $\omega_{n}$ is the wave frequency, and $\varphi_{n}=2 \pi r_{n}$ is an arbitrary but constant phase angle with $r_{n}$ being a random number in the interval $[0,1]$ (Ulmschneider \& Musielak 1998). The velocity amplitudes $u_{n}$ are related to $v_{x}$ and the rms turbulent velocity fluctuations, $u_{\mathrm{t}}$, by

$\overline{v_{x}^{2}}=u_{\mathrm{t}}^{2}=\frac{1}{2} \sum_{n=1}^{N} u_{n}^{2}$,

and they are calculated from

$u_{n}=\sqrt{\frac{4}{3} E^{\prime}\left(\omega_{n}\right) \Delta \omega}$,

where

$E^{\prime}\left(\omega_{n}\right)=\int_{0}^{\infty} E(k) G\left(\frac{\omega_{n}}{k u_{k}}\right) \mathrm{d} k$,
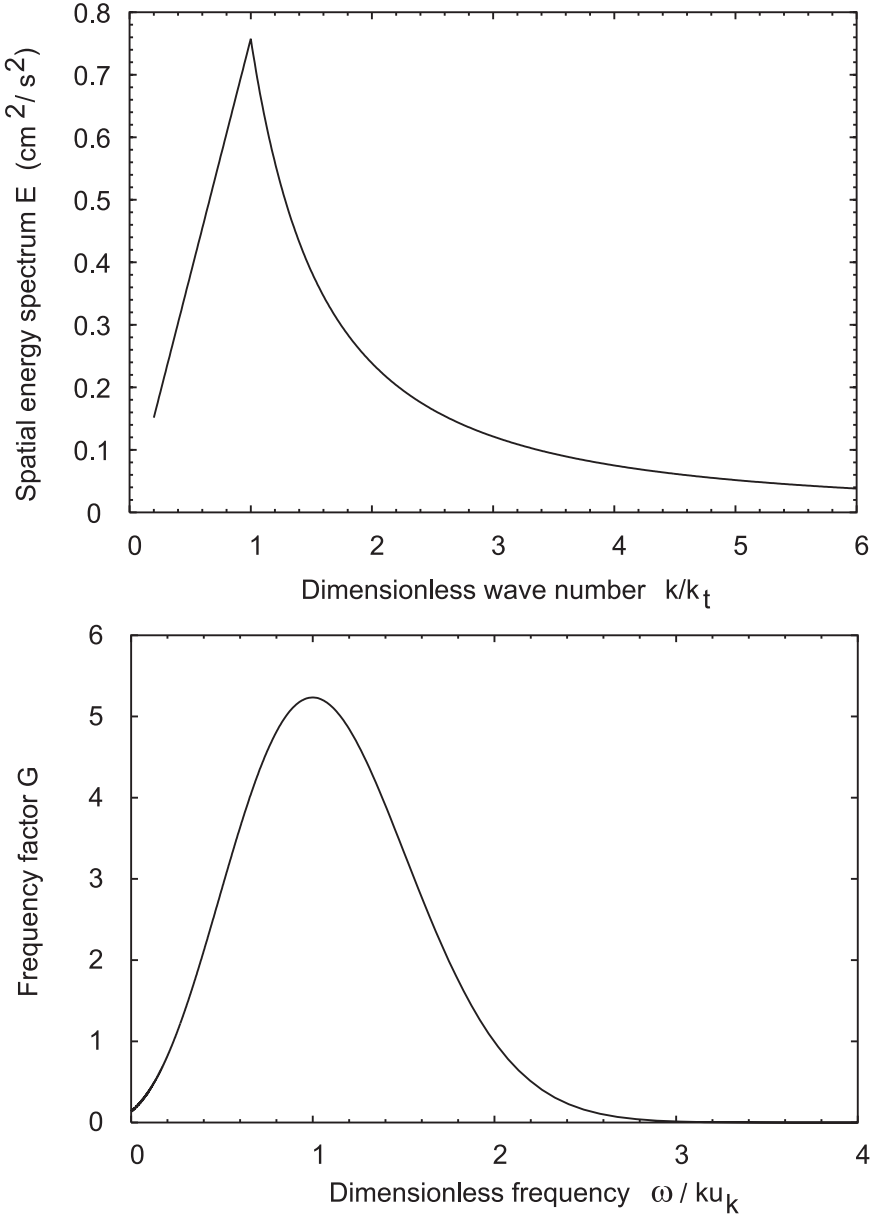

Fig. 3. Extended Kolmogorov spatial turbulent energy spectrum $E$ as a function of the dimensionless wave number $k / k_{\mathrm{t}}$ (top), and modified Gaussian turbulent frequency factor $G$ is plotted versus the dimensionless frequency $\omega / k u_{k}$ (bottom).

and where $E(k)$ and $G\left(\frac{\omega}{k u_{k}}\right)$ represent an extended Kolmogorov spatial turbulent energy spectrum and a modified Gaussian frequency factor, respectively. The reason for this choice of $E$ and $G$ is discussed below. Musielak et al. (1994) have argued that the spatial turbulent energy spectrum must be of the Kolmogorov type and that the main energy carrying bubbles should have a (vertical) size of the local scale height. In reality, there must be some modification of the Kolmogorov law to allow for bubbles larger than this characteristic scale. Since larger bubbles rise from deeper layers where the scale height is larger, it is increasingly more difficult for them to penetrate to the surface. This means that for bubbles with sizes larger than the scale height, the turbulent energy spectrum should decrease. Based on these physical arguments, they proposed that $E(k)$ should be given by

$E(k)= \begin{cases}0 & 0<k<a k_{\mathrm{t}} \\ b \frac{u_{\mathrm{t}}^{2}}{k_{\mathrm{t}}}\left(\frac{k}{k_{\mathrm{t}}}\right) & a k_{\mathrm{t}} \leq k<k_{\mathrm{t}} \\ b \frac{u_{\mathrm{t}}^{2}}{k_{\mathrm{t}}}\left(\frac{k}{k_{\mathrm{t}}}\right)^{-5 / 3} & k_{\mathrm{t}} \leq k \leq k_{\mathrm{d}}\end{cases}$ 
and that $a=0.2$ and $b=0.758$ for the so-called extended Kolmogorov turbulent energy spectrum. Here $k_{\mathrm{t}}$ and $k_{\mathrm{d}}$ are characteristic wave numbers, which will be specified in a moment. To study the effect of the turbulent energy spectrum on the sound generation, these authors have also discussed two other spectra, namely, the broadened and raised Kolmogorov spectra. Note that in both of these spectra the energy in the largest bubbles is artificially increased. It was found that the total acoustic energy did not change much (approximately by a factor of 2 ) but, as expected, the contributions to the high frequency acoustic power were lowered.

In our present work, we take the extended Kolmogorov spectrum (see Eq. (5) and Fig. 3) with a linear rise from $k / k_{\mathrm{t}}=a=0.2$ to 1 , where $k_{\mathrm{t}}=2 \pi / H_{\text {surf }}$ and $H_{\text {surf }}=$ $\Re T_{\text {eff }} /(\mu \mathrm{g})$, with $\Re$ being the gas constant and a molecular weight $\mu=1.3$. Based on this assumption, the largest bubbles have the vertical size $H_{\text {surf }} / a=5 H_{\text {surf }}$. Note that in Eq. (5), $k_{\mathrm{d}}$ is the wave number at which the turbulent cascade ends and that $k_{\mathrm{d}}=2 \pi / l_{\mathrm{d}}$ can be estimated by taking $l_{\mathrm{d}} \approx 4 \mathrm{~cm}$ (e.g., Theurer et al. 1997).

The modified Gaussian frequency factor is given by

$G\left(\frac{\omega}{k u_{k}}\right)=\frac{4}{\sqrt{\pi}} \frac{\omega^{2}}{\left|k u_{k}\right|^{3}} \mathrm{e}^{-\left(\frac{\omega}{k u_{k}}\right)^{2}}$,

and displayed in Fig. 3, where $u_{k}$ is computed from

$u_{k}=\left[\int_{k}^{2 k} E\left(k^{\prime}\right) \mathrm{d} k^{\prime}\right]^{1 / 2}$.

According to Musielak et al. (1994), this form of the frequency factor $G$ is chosen because it has a maximum contribution at $\omega=k u_{k}$. From a physical point of view, this means that one gets maximum correlation when a bubble of gas with size $\lambda=2 \pi / k$, moving with velocity $u_{k}$, has just traveled by a distance equal to its own diameter at a time $t=2 \pi / \omega=\lambda / u_{k}=2 \pi /\left(k u_{k}\right)$. The Gaussian shape is assumed to account for the fact that most (but not all) bubbles have similar lifetimes (see Fig. 3).

The velocity fluctuations $v_{x}(t)$ cause turbulent pressure fluctuations $p_{\text {turb }}^{\prime}$ which are responsible for the squeezing of the flux tube. As shown by Ulmschneider \& Musielak (1998), these fluctuations consist of a time averaged term $\overline{p_{\text {turb }}}=3 \rho_{\mathrm{e}} u_{\mathrm{t}}^{2}$, which augments the external gas pressure $p_{\mathrm{e}}$, and a fluctuating term $p_{\text {turb }}=3 \rho_{\mathrm{e}} v_{x}^{2}(t)$, which gives rise to longitudinal tube waves; note that $\rho_{\mathrm{e}}$ is the gas density in the external medium. Combining these two terms, we get

$p_{\text {turb }}^{\prime}=p_{\text {turb }}-\overline{p_{\text {turb }}}=3 \rho_{\mathrm{e}}\left(v_{x}^{2}-u_{\mathrm{t}}^{2}\right)$.

These turbulent pressure fluctuations lead to gas pressure perturbations $p^{\prime}$ inside the tube

$p^{\prime}=\frac{\beta}{2 / \gamma+\beta} p_{\text {turb }}^{\prime}$, where $\beta=8 \pi p_{0} / B_{0}^{2}$ is the plasma $\beta$. Finally, the gas pressure fluctuations inside the tube can be translated into internal longitudinal velocity fluctuations

$v_{\|}=\frac{c_{\mathrm{S}}^{2}}{c_{\mathrm{T}}} \frac{p^{\prime}}{\gamma p_{0}}$

where $c_{\mathrm{S}}$ is the sound speed, $c_{\mathrm{T}}=\left(1 / c_{\mathrm{S}}^{2}+1 / c_{\mathrm{A}}^{2}\right)^{-1 / 2}$ the tube speed, $c_{\mathrm{A}}$ the Alfvén velocity, $\gamma$ the ratio of specific heats, and $p_{0}$ the gas pressure inside the tube.

Using $\gamma p_{0}=\rho_{0} c_{\mathrm{S}}^{2}$, where $\rho_{0}$ is the density inside the tube, we find that the velocity $v_{\|}$can be written as (Ulmschneider \& Musielak 1998)

$v_{\|}=\frac{\beta}{2 / \gamma+\beta} \frac{3 \rho_{\mathrm{e}}\left(v_{x}^{2}-u_{\mathrm{t}}^{2}\right)}{\rho_{0} c_{\mathrm{T}}}$.

The derived $v_{\|}$is used as the velocity boundary condition in our MHD-wave code. As mentioned above, this boundary condition is applied at the height $z=0$, where in the stellar atmosphere outside the tube one has the optical depth $\tau_{5000}=1$.

At this point one might worry that specifying $v_{\|}$using Eq. (11) is inconsistent with the phase of $p^{\prime}$ derived from $p_{\text {turb }}^{\prime}$. However, we are not interested in the particular phase of the external pressure fluctuation but only in its magnitude and time variation which is translated via Eq. (11) into magnitude and time variation of $v_{\|}$. The hydrodynamic code then takes care of phaseshifts between $p^{\prime}$ and $v_{\|}$.

Specifying $v_{\|}(z=0, t)$ at height $z=0$, where the tube is squeezed, we use our time-dependent, magnetohydrodynamic Lagrangian code (Herbold et al. 1985) to calculate the instantaneous internal pressure perturbation $p^{\prime}(z=0, t)$. Then, these two variables are used to calculate the instantaneous wave energy flux, $F(z, t)=$ $v_{\|}(z, t) p^{\prime}(z, t)$ and the time-averaged wave energy flux, $\overline{F(z, t)}$, also at the height $z=0$. The generated flux represents a mixture of propagating and non-propagating waves. Since we are only interested in propagating waves, we separate these waves from the mixture (see Sect. 3.1) and calculate the propagating wave energy fluxes (see Sect. 3.3).

It is important to note that our code accounts for phase differences between $v_{\|}$and $p^{\prime}$ and that our computations are adiabatic. In addition, our computational domain is only a small section of the tube and the generated waves have relatively small amplitudes. As a result, the process of shock formation does not take place in our calculations. Thus

$F_{\mathrm{L}} \equiv \overline{F(z=0, t)}=\overline{v_{\|}(0, t) p^{\prime}(0, t)}$

represents the wave energy flux carried by longitudinal tube waves in a stellar atmosphere normalized to the stellar surface. The wave energy spectra are computed by performing Fourier transforms of the obtained instantaneous wave energy fluxes. 

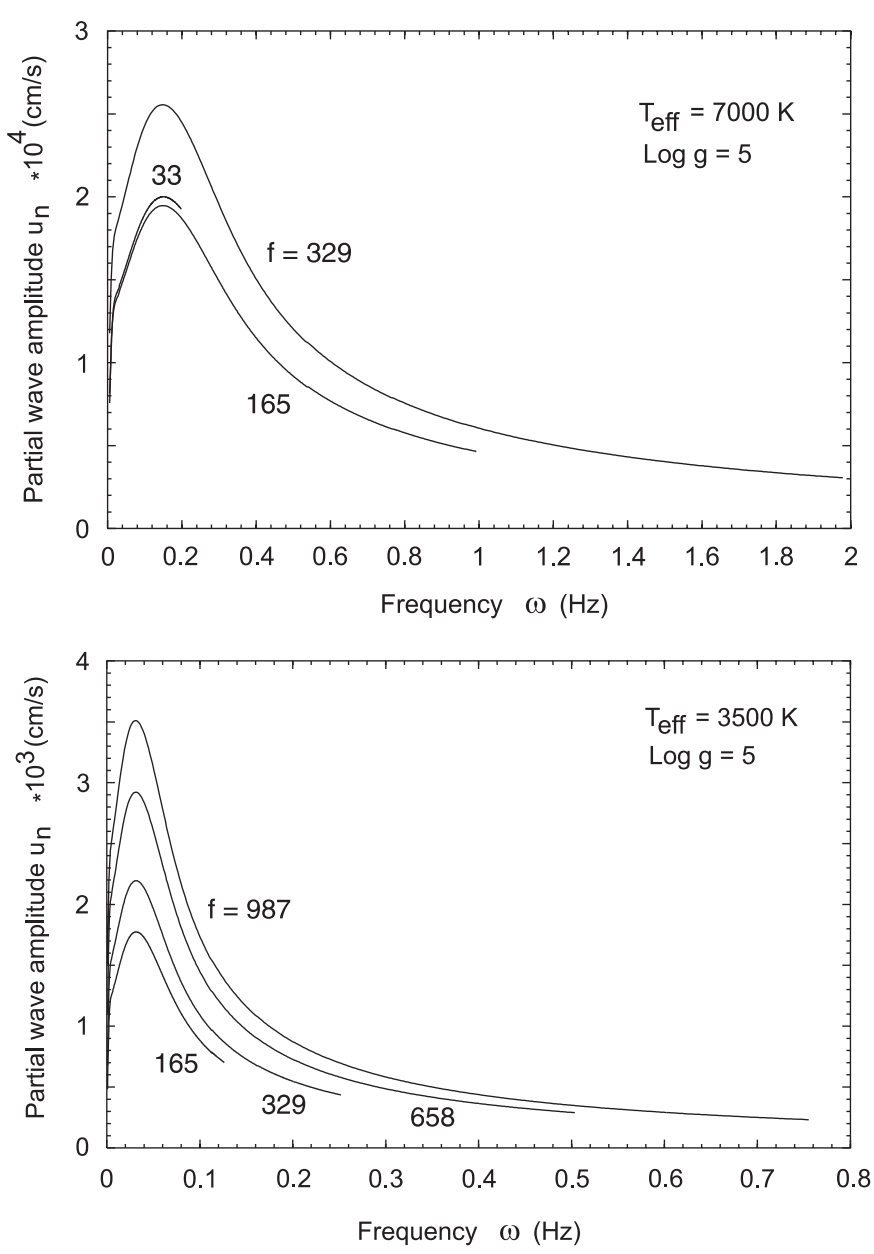

Fig. 4. Spectrum of partial wave amplitudes $u_{n}$ as function of frequency $\omega$ for various ranges $\omega_{1}<\omega<\omega_{N}$ depending on the factor $f=\omega_{N} / \omega_{1}$ for stars of $T_{\text {eff }}=7000 \mathrm{~K}$ (top) and $3500 \mathrm{~K}$ (bottom).

\subsection{Frequency and wave number limits}

The set of frequencies for the partial waves in Eq. (1) require lower, $\omega_{1}$, and upper, $\omega_{N}$, frequency limits. We assume that the lower limit is determined by the size of the largest convective bubbles which come from the depth $z_{\mathrm{b}}$, where $H\left(z_{\mathrm{b}}\right)=H_{\text {surf }} / a=5 H_{\text {surf }}$. Hence, $\omega_{1}=k_{\mathrm{b}} u_{\mathrm{b}}$ with $k_{\mathrm{b}}=2 \pi / H\left(z_{\mathrm{b}}\right)=2 \pi /\left(5 H_{\mathrm{surf}}\right)$. To determine the velocity $u_{\mathrm{b}}$ of these bubbles, we further assume that the form of the turbulent energy spectrum, $E(k)$, at the depth $z_{\mathrm{b}}$ is the same as that given by Eq. (5) but with $u_{\mathrm{t}}\left(z_{\mathrm{b}}\right)=v_{\text {conv }}\left(z_{\mathrm{b}}\right)$, where $v_{\text {conv }}\left(z_{\mathrm{b}}\right)$ is the convective velocity calculated from the mixing-length model at the depth $z_{\mathrm{b}}$. Since the wavenumber at $z_{\mathrm{b}}$ for these bubbles is $k / k_{\mathrm{t}}\left(z_{\mathrm{b}}\right)=1$, we use Eq. (7) to find that the velocity of the largest bubbles at depth $z_{\mathrm{b}}$ is given by $u_{\mathrm{b}}=\sqrt{3 b\left(1-2^{-2 / 3}\right) / 2} v_{\text {conv }}\left(z_{\mathrm{b}}\right)=0.649 v_{\text {conv }}\left(z_{\mathrm{b}}\right)$.

To establish the upper frequency limit, we computed the velocity amplitudes of the partial waves $u_{n}$ by choosing various factors $f$ such that $\omega_{N}=f \omega_{1}$. The results of these calculations obtained for stars with $T_{\text {eff }}=3500 \mathrm{~K}$ and $7000 \mathrm{~K}$ and with $\log g=5$ are presented in Fig. 4, which

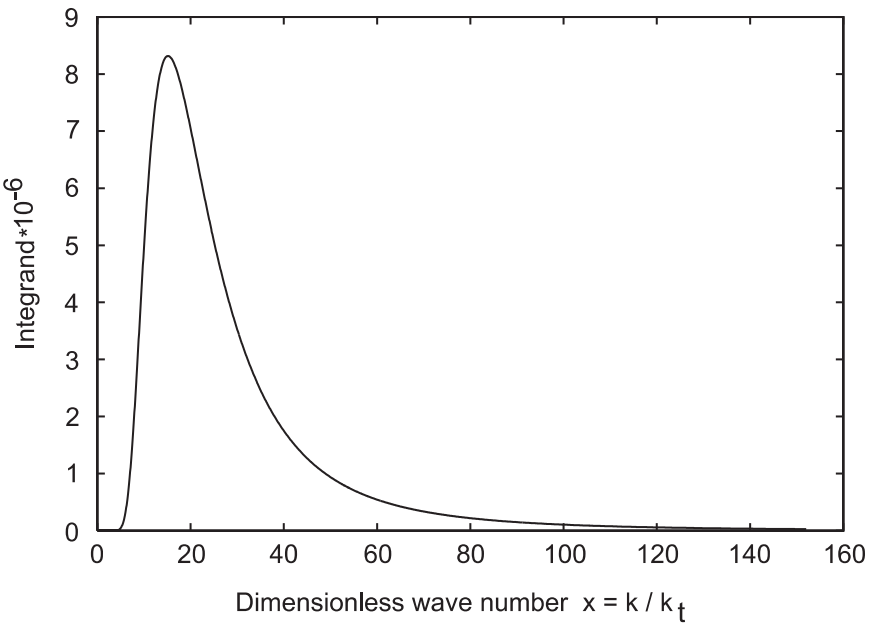

Fig. 5. Behavior of the integrand of Eq. (13) as a function of the dimensionless wave number $x$ for a given partial wave frequency $\omega_{n}$.

shows the range of $u_{n}$ as function of frequency for various factors $f$. It is seen that the velocity amplitudes peak at a certain frequency (these peaks arise from the dominant temporal correlation in the frequency factor $G$ ) and that the factor $f$ must be large enough (300 or more) to resolve the entire spectrum. To ensure that the peak is retained in our computations, we select $f=329$ and use $N=500$ equidistant frequency points $\omega_{n}$.

Another computational parameter, which affects our method of wave generation, is the maximum value of the wave number $k_{\text {Max }}$ used in evaluating the integral given by Eq. (4). As discussed above, one could take $k_{\mathrm{Max}}=k_{\mathrm{d}}$. In practice, a much smaller value is taken. Using the notation $E^{*}(x)=E(k) k_{\mathrm{t}} / u_{\mathrm{t}}^{2}$ and $u_{k}^{*}(x)=u_{k}(k) / u_{\mathrm{t}}$, with $x=k / k_{\mathrm{t}}$, Eq. (4) can be written as

$E^{\prime}\left(\omega_{n}\right)=\frac{4 \omega_{n}^{2}}{\sqrt{\pi} k_{\mathrm{t}}^{3} u_{\mathrm{t}}} \int_{0}^{\infty} \frac{E^{*}(x) \mathrm{e}^{\frac{-\left(\omega_{n} / u_{\mathrm{t}}\right)^{2}}{k_{\mathrm{t}}^{2} x^{2} u_{k}^{*}(x)^{2}}}}{x^{3} u_{k}^{*}(x)^{3}} \mathrm{~d} x$.

For a given value of $\omega_{n}$, as shown in Fig. 5, we find that the integrand of this equation has a pronounced peak at some value $x_{\mathrm{Max}}$ which is caused by the frequency factor $G$, and that after this peak the integrand drops rapidly, due to the exponential behavior of $G$. In order to ensure that the integration captures the main contribution, we determine $x_{\mathrm{Max}}=k_{\mathrm{Max}} / k_{\mathrm{t}}$ for each $\omega_{n}$ and integrate from 0.2 to $10 x_{\max }$ using 2000 points.

\subsection{Defouw cutoff frequency}

The generation and propagation of longitudinal tube waves is affected by the cutoff frequency for these waves. Defouw (1976) was first to show that the cutoff is defined by

$\omega_{\mathrm{D}}=2 \pi \nu_{\mathrm{D}}=\frac{c_{\mathrm{T}}}{H} \sqrt{\frac{9}{16}-\frac{1}{2 \gamma}+\frac{c_{\mathrm{S}}^{2}}{c_{\mathrm{A}}^{2}} \frac{(\gamma-1)}{\gamma^{2}}}$, 


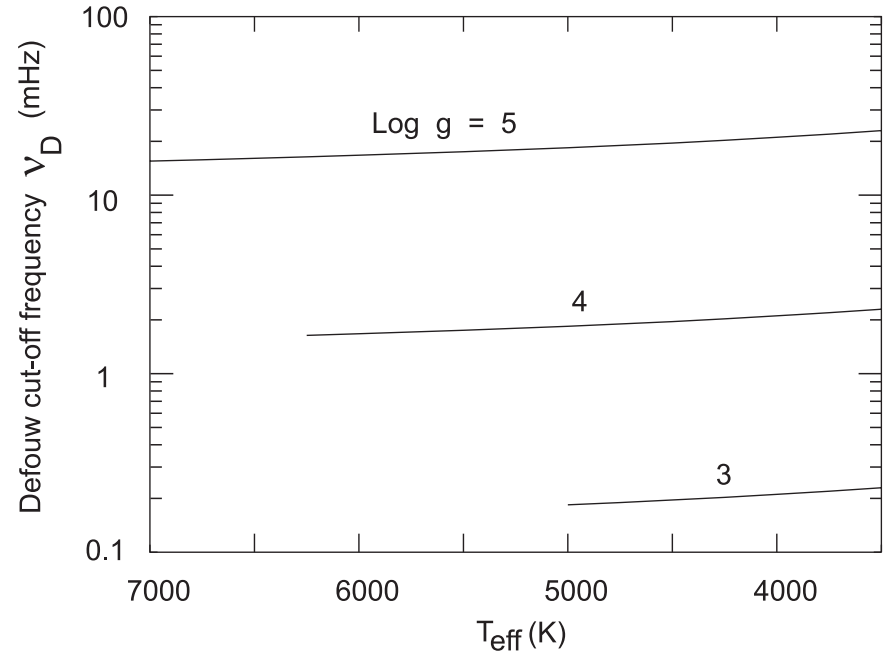

Fig. 6. Defouw cut-off frequencies for stars of different surface gravity $\log g$ and effective temperature $T_{\text {eff }}$. There is very little dependence on the magnetic field strength $B$.

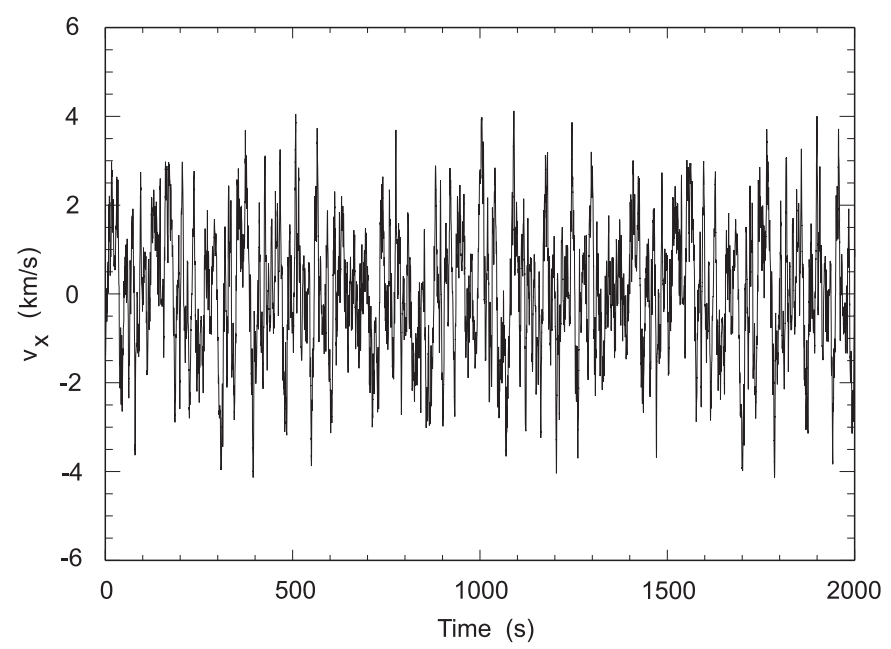

Fig. 7. Instantaneous velocity $v_{x}$ outside a magnetic flux tube with field strength $B=0.85 B_{\text {eq }}$ at height $z=0$ for a star with surface gravity $\log g=5$ and effective temperature $T_{\text {eff }}=$ $6500 \mathrm{~K}$.

where $H$ is the local pressure scale height, and that only waves with frequencies higher than this cutoff are propagating waves. Figure 6 shows the Defouw frequency $\nu_{\mathrm{D}}$ for stars of $T_{\text {eff }}=3500$ to $7000 \mathrm{~K}, \log g=3$ to 5 and different magnetic field strengths $B=0.75,0.85,0.95 B_{\text {eq }}$. One actually finds that the dependence on $B$ is very small and the values are the same within the plotting accuracy.

\section{Results and discussion}

\subsection{Velocity power spectra and propagating wave fluxes}

Using the specified frequency limits we have calculated spectra $u_{n}$ of partial waves for stars of different effective temperatures $T_{\text {eff }}$ and gravities $g$ (two examples of such

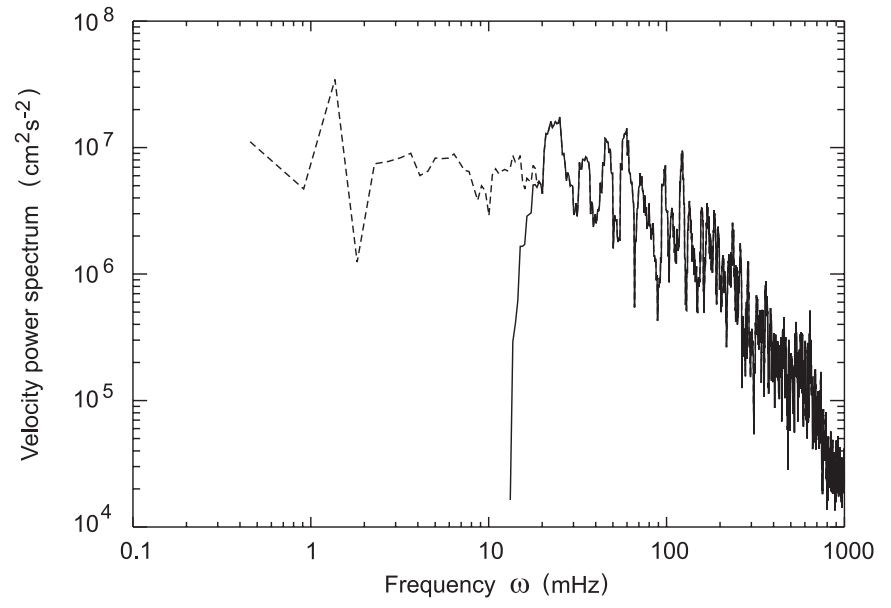

Fig. 8. Velocity power spectrum of the propagating component (solid) and the total power spectrum (dashed) for the star with $T_{\text {eff }}=6500 K, \log g=5$ and $B=0.85 B_{\text {eq }}$.

spectra are shown in Fig. 4). With a stellar atmosphere code, assuming a magnetic field strength given by a fraction of the tube equipartition magnetic field $B_{\text {eq }}$, magnetic tube models are calculated for the considered stars. The spectra $u_{n}$ are then used to compute the turbulent velocity fluctuations $v_{x}(z, t)$ at the squeezing height $z=0$, which corresponds to the optical depth $\tau_{5000}=1$ outside the tube. In Fig. 7, we show this fluctuating turbulent velocity $v_{x}$, as a function of time $t$ up to $t=2000 \mathrm{~s}$ for a star with $T_{\text {eff }}=6500 \mathrm{~K}$ and $\log g=5$, where we have $u_{\mathrm{t}}=1.45 \times 10^{5} \mathrm{~cm} / \mathrm{s}, \omega_{1}=13.2 \mathrm{mHz}, \omega_{N}=4.35 \mathrm{~Hz}$. Due to the many partial waves with random phases, $v_{x}$ is very stochastic in nature which indicates the chaotic character of the prescribed motions as expected for turbulent velocity fluctuations. Using Eq. (11) the longitudinal velocities $v_{\|}$inside the tube can then be computed. We now employ a time-dependent longitudinal wave code using $v_{\|}$as boundary condition and compute the pressure perturbation $p^{\prime}$. To avoid switch-on effects and to allow sufficient time for averaging, these wave computations are extended up to $35 P_{\mathrm{D}}$, where $P_{\mathrm{D}}=1 / \nu_{\mathrm{D}}$ is the Defouw cutoff period (see Sect. 2.5).

The waves generated in the tube represent a mixture of propagating (with frequencies higher than the Defouw cutoff frequency, $\omega_{\mathrm{D}}$ ) and non-propagating (with frequencies lower than $\omega_{\mathrm{D}}$ ) longitudinal tube waves. The reason for this is that both the turbulent energy spectrum and the frequency factor of the fluid motions in the nonmagnetic flow field outside the tube are independent from the cutoff frequency of the waves inside the tube. Hence, the total velocity perturbations $v_{\|}$generated in the tube by the outside pressure perturbations $p_{\text {turb }}^{\prime}$ depend on a set of frequencies that are both above and below the Defouw cutoff frequency. Since we are only interested in propagating waves, the non-propagating wave perturbations have to be removed. To achieve this, we Fourier transform the time-dependent velocity fluctuations $v_{\|}$into the frequency domain $\left(v_{\|}(\omega)=\mathcal{F} v_{\|}(t)\right.$, with suitable apodising at the 
beginning and end of the time string as well as removing nonzero time-averages) and compute the corresponding power spectrum, $P_{\mathrm{v}}(\omega)=v_{\|}^{2}(\omega)$. The result is represented in Fig. 8 by a dashed line which at high frequency is identical to the solid line. It is seen that $v_{\|}^{2}(\omega)$ does not show isolated frequency contributions but only broad noise.

To remove the non-propagating component, we introduce a Heaviside step function, $\mathcal{H}(\omega)$, which is zero below, and one above, the Defouw frequency $\omega_{\mathrm{D}}$ and is subsequently smoothed over 5 grid points in order to prevent the generation of spurious high-frequency noise. For the case considered in Fig. 7, one has a Defouw frequency of $\nu_{\mathrm{D}}=\omega_{\mathrm{D}} /(2 \pi)=16.0 \mathrm{mHz}$, which corresponds to a period $P_{\mathrm{D}}=62.6 \mathrm{~s}$, close to the acoustic cut-off period $P_{\mathrm{A}}=$ $4 \pi c_{\mathrm{S}} /(\gamma g)=63.5 \mathrm{~s}$. Applying the inverse Fourier transform to the product $v_{\|}(\omega) \mathcal{H}(\omega)$ gives the propagating longitudinal velocity component $v_{\|}(t)=\mathcal{F}^{-1}\left(v_{\|}(\omega) \mathcal{H}(\omega)\right)$, which is displayed in Fig. 9; its power spectrum is shown (solid line) in Fig. 8. Comparing $v_{x}$ with $v_{\|}$in Figs. 7 and 9 it is seen that the fluctuations of $v_{x}$ are symmetric whereas the longitudinal velocity $v_{\|}$is very one-sided, although the time-average of these velocity fluctuations is always zero. This one-sidedness, as already discussed by Ulmschneider \& Musielak (1998), is a consequence of Eq. (11).

Now, the same procedure is applied to the pressure fluctuations, $p^{\prime}(t)$. The filtered propagating velocity and pressure fluctuations, $v_{\|}(t)$ and $p^{\prime}(t)$, are then multiplied (see Eq. (12)) and the instantaneous energy flux for propagating longitudinal tube waves is calculated. By removing the negative values of this instantaneous flux, finally the upward propagating longitudinal wave energy flux is obtained. Two examples of the upward propagating fluxes computed for stars with $T_{\text {eff }}=6500 \mathrm{~K}$ and $4000 \mathrm{~K}$ are presented in Figs. 10 and 11. They show the highly stochastic nature of the wave generation. Comparison of Figs. 9 and 10 demonstrates that every spike in the longitudinal wave flux corresponds to a spike in $v_{\|}(t)$. These spikes are caused by large rapidly moving eddies, which according to the Kolmogorov law, have velocities $u_{k} \sim l_{k}^{1 / 3}$. Mathematically, the spikes are described here as a random process of constructive interference between some of the partial waves.

\subsection{Wave power spectra}

We have also computed wave power spectra for each instantaneous wave energy flux. Some of these spectra are displayed in Fig. 12, and are for stars with $T_{\text {eff }}=4000$, $5000,6000,7000 \mathrm{~K}, \log g=5$ and $B=0.85 B_{\text {eq. The }}$ low frequency cut-off (at the Defouw frequency) is due to our removal of the non-propagating part of the velocity and pressure fluctuations (see Fig. 8). The presented spectra also show changes of the Defouw frequency $\omega_{\mathrm{D}}$ with $T_{\text {eff }}$; these changes are consistent with the results shown in Fig. 6. It is seen that the spectra reach their maxima at roughly $\omega=2 \omega_{\mathrm{D}}$ and then decrease relatively slowly

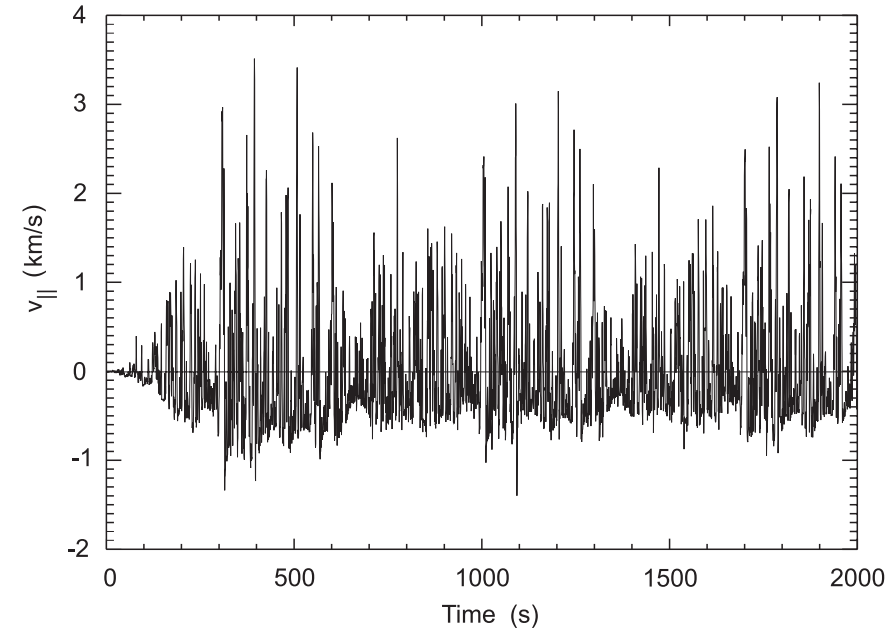

Fig. 9. Instantaneous (apodised) velocity $v_{\|}$of the propagating wave component in the magnetic flux tube with field strength $B=0.85 B_{\text {eq }}$ at height $z=0$ for a star with surface gravity $\log g=5$ and effective temperature $T_{\text {eff }}=6500 \mathrm{~K}$.

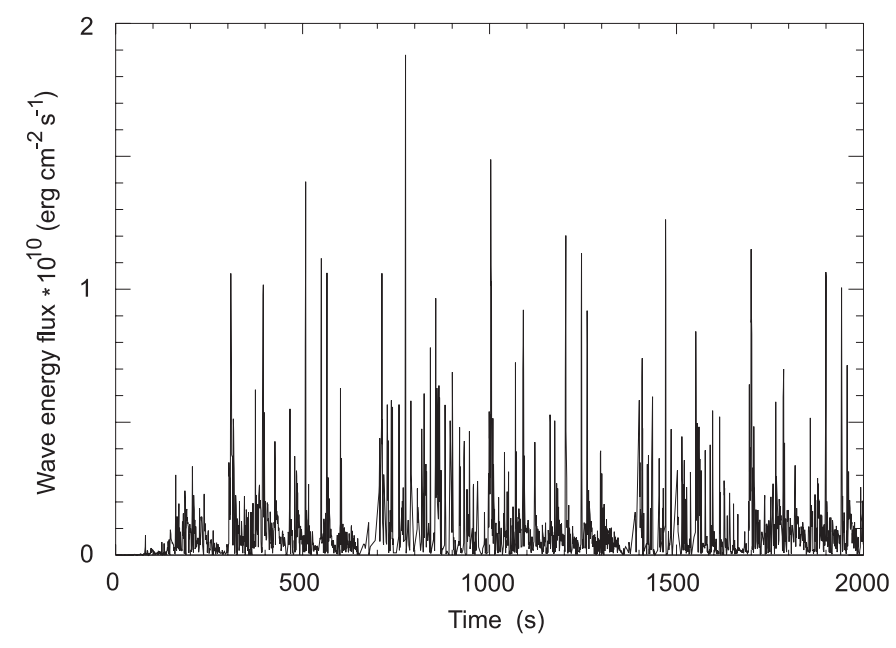

Fig. 10. Instantaneous upward longitudinal tube wave energy flux resulting from the velocity fluctuations shown in Fig. 9. The stochastic nature of the wave generation is shown.

toward higher frequencies. For a two orders of magnitude increase in frequency the power decreases by roughly two orders of magnitude except for the coolest star where the power decreases more rapidly. This slow decrease at the high frequency limit of the power spectrum is another result of the very stochastic nature and the spikyness of the wave generation process, which produces a large number of high frequency events. Moreover, it has to be noted that the power in Fig. 12 actually represents $v_{\|}^{4}$ which indicates that $\mathrm{d} F_{\mathrm{L}}(\omega) / \mathrm{d} \omega \sim \mathrm{d} v_{\|}^{2}(\omega) / \mathrm{d} \omega$ decreases even more slowly with frequency.

\subsection{Time-averaged upward wave energy fluxes}

Due to this spikyness of the instantaneous wave energy fluxes (see Figs. 10 and 11), it is clear that the averaging 


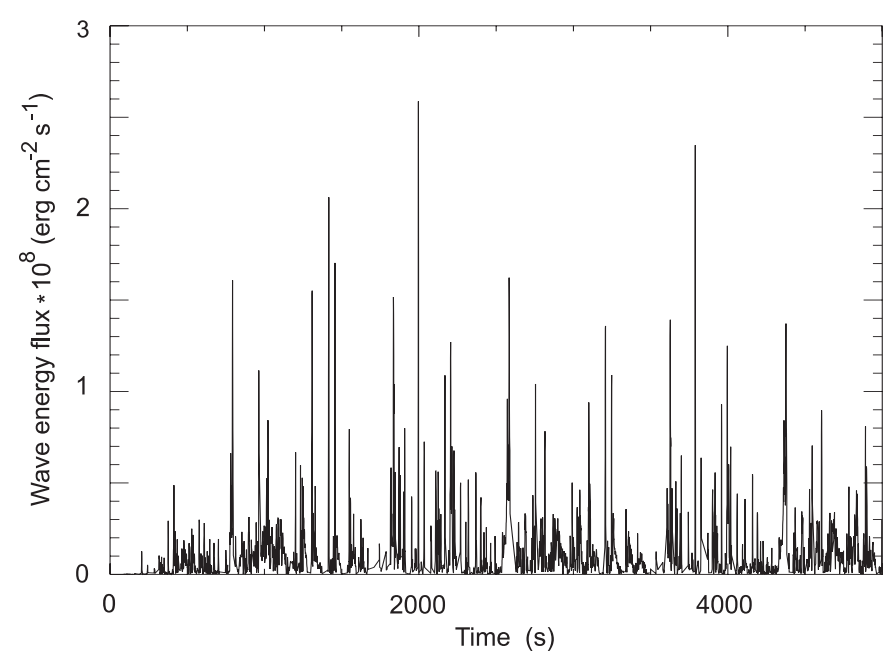

Fig. 11. Instantaneous upward longitudinal tube wave energy flux for a flux tube with $B_{0} / B_{\text {eq }}=0.85$ embedded in an atmosphere of a star with $T_{\text {eff }}=4000 \mathrm{~K}$ and $\log g=5$. The stochastic nature of the wave generation is very prominent.

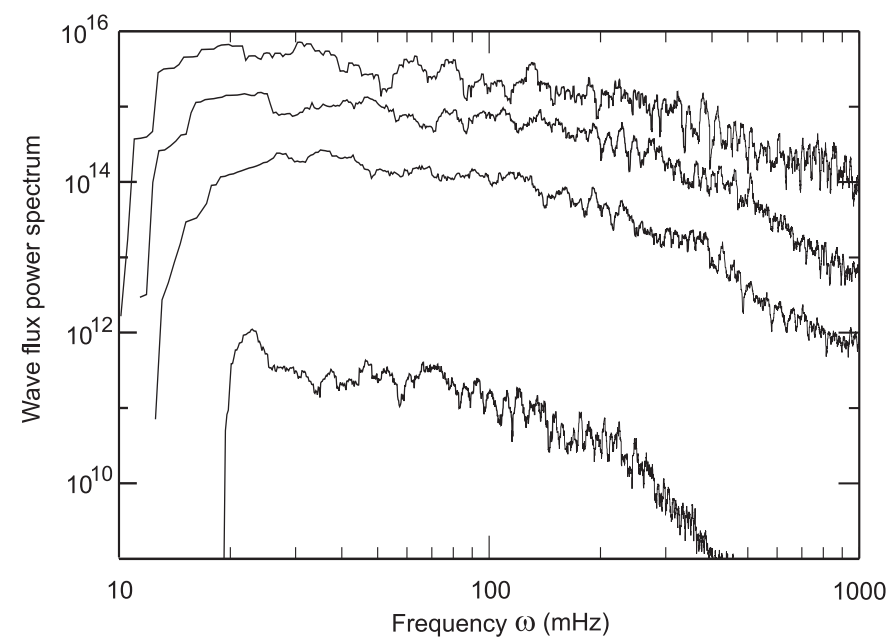

Fig. 12. Power spectra of the instantaneous wave energy flux in magnetic flux tubes with $B_{0} / B_{\text {eq }}=0.85$ embedded in atmospheres of stars with $T_{\text {eff }}=7000,6000,5000,4000 \mathrm{~K}$ and $\log g=5$ as a function of circular frequency $\omega$.

has to be carried out over a long time interval $\left(35 P_{\mathrm{D}}\right)$ to provide reliable average fluxes. The time-averaged longitudinal upward propagating wave energy fluxes, $F_{\mathrm{L}}$, normalized to the stellar surface and computed for magnetic flux tubes embedded in atmospheres of stars with $T_{\text {eff }}$ ranging from 3500 to $7000 K$ and with $\log g$ varying from 3 to 5 , are displayed in Fig. 13 and are also given in Table 1. The values are for flux tubes with magnetic field strengths of $B=0.75,0.85$ and $0.95 B_{\text {eq }}$ at the stellar surface.

Comparing these averaged wave fluxes with the instantaneous fluxes of Figs. 10 and 11, we find that the peaks of the instantaneous fluxes can be orders of magnitude larger than the average flux. For example, one has $F_{\mathrm{L}}=$ $1.0 \times 10^{9} \mathrm{erg} \mathrm{cm}^{-2} \mathrm{~s}^{-1}$ for the star with $T_{\text {eff }}=6500 \mathrm{~K}$, but the spikes can be as high as $1.9 \times 10^{10} \mathrm{erg} \mathrm{cm}^{-2} \mathrm{~s}^{-1}$ (see

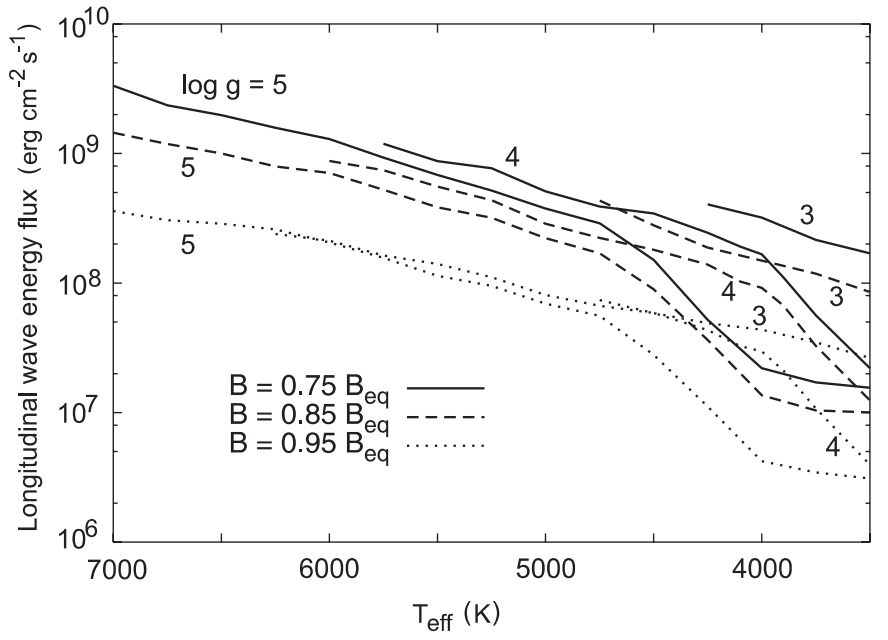

Fig. 13. Time-averaged upward longitudinal wave energy fluxes for late-type stars with different spectral types and luminosities. The strength of the magnetic field $B$ inside stellar flux tubes is a parameter and specified as a fraction the equipartition magnetic field strength, $B_{\text {eq }}$.

Fig. 10); this shows that the wave flux consists essentially of a series of intense short duration bursts. The disparity between the instantaneous and average flux is also present in cool stars. For instance, a star with $T_{\text {eff }}=4000 \mathrm{~K}$ has $F_{\mathrm{L}}=1.4 \times 10^{7} \mathrm{erg} \mathrm{cm}^{-2} \mathrm{~S}^{-1}$ and spikes of the order of $2.6 \times 10^{8} \mathrm{erg} \mathrm{cm}^{-2} \mathrm{~s}^{-1}$ (see Fig. 11). The disparity amounts to a factor of 19 for both stars which clearly indicates that the averaging for cool stars has to be extended to long time intervals to obtain stable average wave fluxes.

The extreme spikyness is in good agreement with observations by Muller (19) and Muller et al. (1994), and with models by Choudhuri et al. (1993a) and (1993b). This peculiar nature of the wave fluctuations shows that it is essential to include nonlinear effects in the longitudinal wave generation.

Figure 13 and Table 1 show that the wave energy generation depends significantly on effective temperature and gravity. This is a consequence of the dependence of the rms velocity $u_{\mathrm{t}}$ on $T_{\text {eff }}$ and $\log g$ displayed in Fig. 2. The explanation of this behavior has already been given by Ulmschneider et al. (1996) in their acoustic wave generation calculations. The main excitation of the magnetic flux tubes by external turbulence occurs near the top of the convection zone where the convection flux, $\rho v_{\text {conv }}^{3}$, is still essentially equal to the total stellar radiative flux, $\sigma T_{\text {eff }}^{4}$, and where the convective velocity $v_{\text {conv }}$ has its maximum. Lowering $T_{\text {eff }}$ at constant $\log$ g thus decreases $v_{\text {conv }}$ and $u_{\mathrm{t}}$, while lowering gravity with $T_{\text {eff }}$ held constant lowers the atmospheric density $\rho$ and increases $v_{\text {conv }}$ and $u_{\mathrm{t}}$ (Figs. 1, 2).

Based on the results presented in Table 1, the average total upward wave energy fluxes obtained for different values of $u_{\mathrm{t}}$ can be approximately fitted by

$F_{\mathrm{L}} \approx 4.3 \times 10^{-3} u_{\mathrm{t}}^{2.2} \mathrm{erg} \mathrm{cm} \mathrm{cm}^{-2} \mathrm{~s}^{-1}$, 
Table 1. Rms turbulent velocities $u_{\mathrm{t}}(\mathrm{cm} / \mathrm{s})$ at the squeezing height and average total fluxes, $F_{\mathrm{L}}\left(\mathrm{erg} \mathrm{cm}^{-2} \mathrm{~s}^{-1}\right)$, for upward propagating longitudinal tube waves in stars with different effective temperatures $T_{\text {eff }}$, gravity $\log g$, and magnetic field strength $B$.

\begin{tabular}{|c|c|c|c|}
\hline$T_{\text {eff }}$ & $\log g=3$ & 4 & 5 \\
\hline & & $u_{\mathrm{t}}(\mathrm{cm} / \mathrm{s})$ & \\
\hline 3500 & $8.2 \times 10^{4}$ & $3.3 \times 10^{4}$ & $1.8 \times 10^{4}$ \\
\hline 4000 & $1.2 \times 10^{5}$ & $7.0 \times 10^{4}$ & $2.8 \times 10^{4}$ \\
\hline 4500 & $1.5 \times 10^{5}$ & $9.7 \times 10^{4}$ & $5.5 \times 10^{4}$ \\
\hline 5000 & $1.8 \times 10^{5}$ & $1.2 \times 10^{5}$ & $7.8 \times 10^{4}$ \\
\hline 5500 & & $1.5 \times 10^{5}$ & $9.6 \times 10^{4}$ \\
\hline 6000 & & $1.8 \times 10^{5}$ & $1.2 \times 10^{5}$ \\
\hline 6500 & & & $1.5 \times 10^{5}$ \\
\hline \multirow[t]{2}{*}{7000} & & & $1.7 \times 10^{5}$ \\
\hline & & $B=0.75 B_{\mathrm{eq}}$ & \\
\hline 3500 & $1.7 \times 10^{8}$ & $2.2 \times 10^{7}$ & $1.6 \times 10^{7}$ \\
\hline 4000 & $3.2 \times 10^{8}$ & $1.7 \times 10^{8}$ & $2.2 \times 10^{7}$ \\
\hline 4500 & & $3.4 \times 10^{8}$ & $1.5 \times 10^{8}$ \\
\hline 5000 & & $5.1 \times 10^{8}$ & $3.8 \times 10^{8}$ \\
\hline 5500 & & $8.7 \times 10^{8}$ & $6.8 \times 10^{8}$ \\
\hline 6000 & & & $1.3 \times 10^{9}$ \\
\hline 6500 & & & $2.0 \times 10^{9}$ \\
\hline \multirow[t]{2}{*}{7000} & & & $3.3 \times 10^{9}$ \\
\hline & & $B=0.85 B_{\text {eq }}$ & \\
\hline 3500 & $8.5 \times 10^{7}$ & $1.2 \times 10^{7}$ & $1.0 \times 10^{7}$ \\
\hline 4000 & $1.5 \times 10^{8}$ & $9.2 \times 10^{7}$ & $1.4 \times 10^{7}$ \\
\hline 4500 & $2.8 \times 10^{8}$ & $1.8 \times 10^{8}$ & $9.0 \times 10^{7}$ \\
\hline 5000 & & $2.9 \times 10^{8}$ & $2.2 \times 10^{8}$ \\
\hline 5500 & & $5.6 \times 10^{8}$ & $3.9 \times 10^{8}$ \\
\hline 6000 & & $8.8 \times 10^{8}$ & $7.1 \times 10^{8}$ \\
\hline 6500 & & & $1.0 \times 10^{9}$ \\
\hline \multirow[t]{2}{*}{7000} & & & $1.4 \times 10^{9}$ \\
\hline & & $B=0.95 B_{\text {eq }}$ & \\
\hline 3500 & $2.6 \times 10^{7}$ & $4.0 \times 10^{6}$ & $3.1 \times 10^{6}$ \\
\hline 4000 & $4.4 \times 10^{7}$ & $3.0 \times 10^{7}$ & $4.2 \times 10^{6}$ \\
\hline 4500 & $5.9 \times 10^{7}$ & $5.9 \times 10^{7}$ & $2.8 \times 10^{7}$ \\
\hline 5000 & & $8.1 \times 10^{7}$ & $6.9 \times 10^{7}$ \\
\hline 5500 & & $1.4 \times 10^{8}$ & $1.1 \times 10^{8}$ \\
\hline 6000 & & $2.1 \times 10^{8}$ & $2.1 \times 10^{8}$ \\
\hline 6500 & & & $2.9 \times 10^{8}$ \\
\hline 7000 & & & $3.6 \times 10^{8}$ \\
\hline
\end{tabular}

for stars with $\log g=5$, and

$F_{\mathrm{L}} \approx 6.1 \times 10^{-5} u_{\mathrm{t}}^{2.5} \mathrm{erg} \mathrm{cm}^{-2} \mathrm{~s}^{-1}$,

for $\log g=4$, where in both cases, it is assumed that $B=$ $0.85 B_{\text {eq. }}$. Both the magnitude of the exponent of $u_{\mathrm{t}}$ and the mode of excitation show that longitudinal tube waves are generated by monopole (or dipole) emission which has already been recognized by Musielak et al. (1989, 1995) in their analytical treatment of the generation of these waves.

The results given in Table 1 also demonstrate that the generated wave energy flux decreases when the strength of the tube magnetic field is increased. This well-known effect has been extensively discussed by Musielak et al. (1989, 1995) and Ulmschneider \& Musielak (1998), and is attributed to the increasing stiffness of magnetic flux tubes when its magnetic field strength increases. With greater field strength, the density inside the tube decreases, and there is less gas to support longitudinal tube waves (see Sect. 3.4, for a further discussion). The approximate relationships describing this effect are

$F_{\mathrm{L}} \approx 4.8 \times 10^{7}\left(B / B_{\mathrm{eq}}\right)^{-7.2} \mathrm{erg} \mathrm{cm}^{-2} \mathrm{~s}^{-1}$,

for stars with $\log g=5$, and

$F_{\mathrm{L}} \approx 5.4 \times 10^{7}\left(B / B_{\mathrm{eq}}\right)^{-7.8} \mathrm{erg} \mathrm{cm}^{-2} \mathrm{~s}^{-1}$,

for $\log g=4$, where in both cases, $T_{\text {eff }}=5000 \mathrm{~K}$. It is seen that the dependence of longitudinal wave energy fluxes on the magnetic field strength in the flux tubes is very similar for stars of the same $T_{\text {eff }}$ and different gravities. An interesting result is, that this dependence on the magnetic field does not change much when stars of different $T_{\text {eff }}$ but similar gravities are considered. This means that the effects caused by the magnetic field on the generation of longitudinal tube waves are very similar in all late-type stars.

\subsection{Comparison to previous results}

The computed wave energy spectra and fluxes are now compared with those obtained previously for longitudinal and transverse tube waves, and also for acoustic waves.

1. In the paper by Ulmschneider \& Musielak (1998), we have used for the Sun the same numerical method as in the present paper and have compared the computed fluxes to those obtained analytically by Musielak et al. (1995). That comparison showed that the numerical fluxes are typically an order of magnitude larger than those computed analytically. The difference can be explained by the fact that the numerical method with its singular excitation point allows for very spiky nonlinear waves and does not take into account the correlation effects due to simultaneous excitation at many height points (see Sect. 2.1). As a result, we tentatively regard the fluxes given by Ulmschneider \& Musielak and those presented here as upper bounds for the amount of longitudinal wave energy generated in the solar and stellar convection zones.

2. Recently, Musielak et al. (2000) have used their analytical method to compute the wave energy spectra and fluxes for longitudinal tube waves generated in convection zones of stars with $T_{\text {eff }}$ ranging from $3000 \mathrm{~K}$ to $8000 \mathrm{~K}$ with gravities $\log g=3$ to 5 . Comparing their results with those in the present paper, we find that the conclusions reached from the comparison of the solar fluxes are also valid for 
Table 2. Average upward longitudinal tube wave fluxes, $F_{\mathrm{L}}\left(\mathrm{erg} \mathrm{cm}^{-2} \mathrm{~s}^{-1}\right)$ for the Sun $\left(T_{\text {eff }}=5770 \mathrm{~K}, g=2.436 \times\right.$ $\left.10^{4} \mathrm{~cm} \mathrm{~s}^{-2}\right)$ in tubes of different magnetic field strengths $B / B_{\text {eq }}$ where $B_{\text {eq }}=1976 \mathrm{G}$.

\begin{tabular}{|l||c|}
\hline$B / B_{\text {eq }}$ & $F_{\mathrm{L}}$ \\
\hline \hline 0.65 & $1.5 \times 10^{9}$ \\
0.75 & $1.2 \times 10^{9}$ \\
0.85 & $6.6 \times 10^{8}$ \\
0.95 & $1.9 \times 10^{8}$ \\
\hline
\end{tabular}

other stars, namely, the numerically calculated fluxes are always higher than those obtained analytically. These differences vary with the effective temperature and gravity. For example in the case $B / B_{\text {eq }}=0.85$, for $\log g=5$ the difference varies from a factor of 20 for $T_{\text {eff }}=7000 \mathrm{~K}$ to a factor of 500 for $T_{\text {eff }}=3500 \mathrm{~K}$, while for $\log g=4$ from a factor 20 for $T_{\text {eff }}=6000 \mathrm{~K}$ to a factor of 200 for $T_{\text {eff }}=3500 \mathrm{~K}$, and for $\log g=3$ from a factor 20 for $T_{\text {eff }}=4750 \mathrm{~K}$ to a factor of 200 for $T_{\text {eff }}=3500 \mathrm{~K}$. In the analytical results the fluxes for a given $T_{\text {eff }}$ in the range of $7000 \mathrm{~K}$ to $3000 \mathrm{~K}$ increases by a factor of 60 for lowering the gravity by each factor 10 . The numerical results vary much less for a similar change of gravity except for the stars with $T_{\text {eff }}=4000 \mathrm{~K}$ and cooler.

The large differences for cool stars can be attributed to the fact that these stars have very low turbulent velocities, which in the analytical approach are mainly responsible for the generation of non-propagating waves (see Musielak et al. 2000 for a detailed discussion). Apparently, more energy is generated as propagating waves in the numerical approach because larger instantaneous velocities are imposed on stellar magnetic flux tubes at the excitation height located at $\tau_{5000}=1$.

Comparing the numerical and analytical longitudinal tube wave fluxes we also find that the latter depend on the magnetic field strength as $\left(B / B_{\text {eq }}\right)^{-9.3}$, while the former as $\left(B / B_{\text {eq }}\right)^{-7.2}$ (see Eq. (17)). This implies that the analytical approach predicts much lower wave energy fluxes when the stiffness of stellar magnetic flux tubes increases (see Sect. 3.3). On the other hand, larger fluxes are obtained by using the numerical method because that method takes the nonlinear effects into account.

3. Important differences are found when comparing our present work with the solar results of Ulmschneider \& Musielak (1998). By taking the upward propagating longitudinal flux in the present work instead of the total propagating flux in the 1998 paper (as suggested by Reiner Hammer 2000, private communication), we gain roughly a factor of two. But there are also differences when comparing our present velocity and magnetic field dependences of Eqs. (15) to (18) with the results of Eq. (27) in the 1998 paper. The fact that there is a different dependence on the rms velocity $u_{\mathrm{t}}$ is not surprising because in our present paper $u_{\mathrm{t}}$ is fixed for a given star. Therefore Eqs. (15) and (16) refer to velocities $u_{\mathrm{t}}$ of stars of different $T_{\text {eff }}$, while in Ulmschneider \& Musielak, the $u_{\mathrm{t}}$-dependence refers to variations of $u_{\mathrm{t}}$ in a single star, the Sun with fixed $T_{\text {eff }}$.

What is more puzzling at first sight is the large difference in the dependence of the longitudinal wave flux on the magnetic field strength $B$. The solar work of Ulmschneider \& Musielak (1998) differs from our present work in the external atmosphere model. While in the former a semiempirical extended VAL model was used, our present work for the large variety of stars employs theoretical radiative equilibrium atmosphere models in which magnetic tubes of various field strengths are embedded. For solar $T_{\text {eff }}$ and gravity, therefore, different external gas pressures, $p_{\mathrm{e}}$ are found at $\tau_{5000}=1$ and consequently different equipartition field strengths $B_{\text {eq }}=\sqrt{8 \pi p_{\mathrm{e}}}$. In the VAL model e.g. one has $B_{\text {eq }}=1716 \mathrm{G}$, while in our present work our solar atmosphere model has $B_{\text {eq }}=1976 \mathrm{G}$. While this difference contributes to the different $B$-dependences, a second effect is even more important.

Consider the upward longitudinal tube wave fluxes for a solar model in Table 2. It is seen that these fluxes saturate for low field strengths and show a strong $B$-variation when the magnetic field strength is high. Actually taking the two $F_{\mathrm{L}}$ values for $B / B_{\text {eq }}=0.85$ and 0.95 , we find for the Sun $F_{\mathrm{L}} \approx 1.0 \times 10^{8}\left(B / B_{\text {eq }}\right)^{-11.4}$, while if we take the values $B / B_{\text {eq }}=0.65$ and 0.75 , we find $F_{\mathrm{L}} \approx 7.6 \times 10^{8}\left(B / B_{\text {eq }}\right)^{-1.5}$. This clearly shows that the longitudinal tube wave fluxes depend strongly on $B$ if $B$ is high, and much less strongly on $B$ when $B$ is low, which explains most of the difference to the results of Ulmschneider \& Musielak (1998).

4. Comparing the longitudinal wave energy fluxes by Ulmschneider \& Musielak (1998) with the transverse wave energy fluxes obtained by Huang et al. (1995) shows that the latter are approximately 30 times higher than the former. Since it is much easier to generate transverse than longitudinal tube waves in stellar convection zones (e.g., Spruit \& Roberts 1983), we expect stellar transverse wave energy fluxes to be significantly higher than the longitudinal fluxes computed in this paper. However, the direct comparison cannot be done presently because nonsolar stellar transverse wave energy fluxes have not been calculated.

5. Finally, we compare our stellar longitudinal wave fluxes with the acoustic wave energy fluxes by Ulmschneider et al. (1996). Note that longitudinal tube waves are essentially one-dimensional acoustic waves, propagating along magnetic flux tubes. For the Sun, Ulmschneider \& Musielak (1998) found that the longitudinal wave energy fluxes are roughly equal to typical solar acoustic wave fluxes. We now find that the Sun, with an acoustic flux of $F_{\mathrm{A}}=1.4 \times 10^{8} \mathrm{erg} \mathrm{cm}^{-2} \mathrm{~s}^{-1}$ has a factor of 5 greater upward longitudinal wave flux $F_{\mathrm{L}}=6.6 \times 10^{8} \mathrm{erg} \mathrm{cm}^{-2} \mathrm{~s}^{-1}$ (see Table 2). The selection of the upward propagating flux instead of the total propagating flux increases the longitudinal wave fluxes in the present work.

For the case $B / B_{\text {eq }}=0.85$ and a mixing length parameter $\alpha=2.0$ we find now for $\log g=5$ that the difference 
between the upward propagating longitudinal tube wave flux and the acoustic flux varies from a factor of 2.0 for $T_{\text {eff }}=7000 \mathrm{~K}$ to a factor of 450 for $T_{\text {eff }}=3500 \mathrm{~K}$, while for $\log g=4$ from a factor 2.0 for $T_{\text {eff }}=6000 \mathrm{~K}$ to a factor of 40 for $T_{\text {eff }}=3500 \mathrm{~K}$, and for $\log g=3$ from a factor 3.0 for $T_{\text {eff }}=4500 \mathrm{~K}$ to a factor of 17 for $T_{\text {eff }}=3500 \mathrm{~K}$. While the longitudinal fluxes for hotter stars are a factor of two larger, these differences become much larger towards cool stars because of the much flatter $T_{\text {eff-dependence of }}$ the magnetic waves. The reason for this behavior is that the acoustic flux has a $u_{\mathrm{t}}^{8}$-dependence due to quadrupole sound generation (Lighthill formula) while the longitudinal tube waves have an $u_{\mathrm{t}}^{2-3}$-dependence (Eqs. (15), (16)) which is due to monopole sound generation.

The comparison clearly shows that the wave energy flux inside a stellar magnetic tube is much higher than the acoustic flux in the external medium. In addition, because the wave in the tube travels in an atmosphere which has a much lower gas density than the outside medium, the higher wave energy will lead to a much stronger heating of these tubes. This enhanced heating can be used to explain the increased chromospheric activity in magnetically active late-type stars.

\section{Conclusions}

From our studies of the nonlinear generation of longitudinal waves in stellar magnetic flux tubes, the following general conclusions can be drawn. As we have been forced to make many critical assumptions on the property of the turbulence and the magnitude of the velocity fluctuations for the different stars we must caution the reader about the reliability of our numerical flux values.

1. Through nonlinear time-dependent response of stellar magnetic flux tubes to continuous and impulsive external turbulent pressure fluctuations, longitudinal tube waves are generated by the process of monopole emission.

2. The shapes of the computed power spectra are similar for stars of different effective temperature and gravity. However, the longitudinal wave flux at a given frequency is much higher for hot than for cool stars.

3. The total upward longitudinal wave energy flux increases both with higher effective temperature and decreasing gravity.

4. The computed wave energy flux depends strongly on the magnetic field strength $B$. For a given spectral type and luminosity class, the flux can be almost one order of magnitude higher in tubes with a field strength of $B / B_{\text {eq }}=0.75$ than in those with $B / B_{\text {eq }}=0.95$. The wave energy fluxes depend strongly on $B$ if $B$ is high, and much less strongly on $B$ when $B$ is low.

5. The longitudinal wave energy fluxes are higher than the acoustic fluxes computed for the same stars. For cool dwarfs, the longitudinal fluxes are much higher. This is due to the $u_{\mathrm{t}}^{8}$ and $u_{\mathrm{t}}^{2-3}$ dependences on the convective velocity for the acoustic and longitudinal tube waves, respectively, and signifies the difference between quadrupole and monopole sound generation. Because the magnetic tubes carrying the longitudinal waves have a lower density, the heating in the tubes is much more dramatic. This can explain the intense heating in magnetic regions.

6. The total upward wave energy fluxes calculated with the numerical method are approximately a factor of 20 higher than those obtained analytically. These differences increase to factors of 200 to 500 for cool dwarfs.

7. The obtained longitudinal wave energy fluxes represent upper bounds for the realistic wave energy fluxes generated in stellar atmospheres.

Acknowledgements. This work was supported by the DFG grant Ul57/25-3, by NATO under grant CRG-910058 (P. U. and Z. E. M.) and NSF under grant ATM-0087184 (Z. E. M. and P. U.). Z. E. M. also acknowledges the support of this work by the Alexander von Humboldt Foundation. We are particularly thankful to Reiner Hammer for pointing out to us as referee that we should take the upward propagating longitudinal wave flux instead of the total propagating flux as we had done in Ulmschneider \& Musielak (1998).

\section{References}

Bohn, H. U. 1981, Ph.D. Thesis, Univ. Würzburg, Germany

Bohn, H. U. 1984, A\&A, 136, 338

Buchholz, B., Ulmschneider, P., \& Cuntz, M. 1998, ApJ, 494, 700

Cattaneo, F., Brummell, N. H., Toomre, J., Malagoli, A., \& Hulburt, N. E. 1991, ApJ, 370, 282

Choudhuri, A. R., Auffret, H., \& Priest, E. R. 1993a, Sol. Phys., 143,49

Choudhuri, A. R., Dikpati, M., \& Banerjee, D. 1993b, ApJ, 413,811

Cuntz, M., Ulmschneider, P., \& Musielak, Z. E. 1998, ApJ, 493, L117

Cuntz, M., Rammacher, W., Ulmschneider, P., Musielak, Z. E., \& Saar, S. H. 1999, ApJ, 522, 1053

Defouw, R. J. 1976, ApJ, 209, 266

Herbold, G., Ulmschneider, P., Spruit, H. C., \& Rosner, R. 1985, A\&A, 145, 157

Huang, P., Musielak, Z. E., \& Ulmschneider, P. 1995, A\&A, 279,579

Hünsch, M., \& Schröder, K.-P. 1997, A\&A, 309, L51

Lighthill, M. J. 1952, Proc. Roy. Soc. London A, 211, 564

Muller, R. 1989, in Solar and Stellar Granulation, ed. R. J. Rutten, \& G. Severino (Kluwer Academic Publ., Dordrecht), 101

Muller, R., Roudier, Th., Vigneau, J., \& Auffret, H. 1994, A\&A, 283, 232

Musielak, Z. E., Rosner, R., \& Ulmschneider, P. 1989, ApJ, 337,470

Musielak, Z. E., Rosner, R., Stein, R. F., \& Ulmschneider, P., 1994, ApJ, 423, 474

Musielak, Z. E., Rosner, R., Gail, H. P., \& Ulmschneider, P., 1995, ApJ, 448, 865

Musielak, Z. E., Rosner, R., \& Ulmschneider, P. 2000, ApJ, 541,410

Narain, U., \& Ulmschneider, P. 1996, Space Sci. Rev., 75, 453

Nesis, A., et al. 1992, ApJ, 399, L99 
Nordlund, A., \& Dravins, D. 1990, A\&A, 238, 155

Nordlund, A., Spruit, H. C., Ludwig, H.-G., \& Trampedach, R. 1997, A\&A, 328, 229

Nordlund, A., \& Stein, R. F. 1991, in Stellar Atmospheres: Beyond Classical Models, ed. L. Crivellari, \& I. Hubeny, NATO ASI Ser. (Kluwer Academic Publ., Dordrecht), 263

Rüedi, I., Solanki, S. K., Mathys, G., \& Saar, S. H. 1997, A\&A, 318,429

Saar, S. H. 1996, in Magnetodynamic Phenomena in the Solar Atmosphere - Prototypes of Stellar Magnetic Activity, IAU Coll. 153, ed. Y. Uchida, et al. (Dordrecht: Kluwer), 367

Schröder, K.-P., \& Eggleton, P. P. 1996, Rev. Mod. Astr., 9, 221

Solanki, S. K. 1993, Space Sci. Rev., 63, 1

Spruit, H. C., \& Roberts, B. 1983, Nature, 304, 401

Steffen, M. 1993, Habil. Thesis, Univ. Kiel, Germany

Stein, R. F. 1967, Sol. Phys., 2, 385
Stenflo, J. O. 1978, Rep. Progr. Phys., 75, 3

Theurer, J., Ulmschneider, P., \& Kalkofen, W. 1997, A\&A, 324,717

Trampedach, R., Christensen-Dalsgaard, J., Nordlund, A., \& Stein, R. F. 1997, in Solar Convection and Oscillations and their Realationship, ed. F. P. Pijpers, J. ChristensenDalsgaard, \& C. S. Rosenthal (Kluwer Academic Publishers), 73

Ulmschneider, P., Theurer, J., \& Musielak, Z. E. 1996, A\&A, 315,212

Ulmschneider, P., Theurer, J., Musielak, Z. E., \& Kurucz, R. 1999, A\&A, 347, 243

Ulmschneider, P., \& Musielak, Z. E. 1998, A\&A, 338, 311

Ulmschneider, P., Zähringer, K., \& Musielak, Z. E. 1991, A\&A, 241,625

Vernazza, J. E., Avrett, E. H., \& Loeser, R. 1981, ApJS, 45, 635 